\title{
A COMPARISON OF THE WASSERMANN AND MEINICKE (M.K.R. II) TESTS IN THE SEROLOGICAL DIAGNOSIS OF SYPHILIS *
}

By M. M. BARRITT, M.Sc., Dp.Bact., L.M.S.S.A. (First Assistant City Bacteriologist, Liverpool Corporation); with a Note by A. O. ROSS, M.D., D.P.H. (Chief Venereal Diseases Medical Officer, Liverpool Corporation).

\section{Methods Used \\ I. The Wassermann Test (W.R.)}

THIS was the No. I method of Harrison described in the "Medical Research Committee Special Report Series, No. I4," London (I9r8), and modified by Wyler (I929); it requires the use of a constant volume of patient's serum ( $I: 5$ dilution) and a quantitative estimation of the strength of the reactions by means of 3 and 5 minimal hæmolytic doses (m.h.d.) of guinea-pig complement. The original method was employed but minor modifications introduced were the use of sheep heart antigen (Burroughs Wellcome \& Co., London) and the mixing of complement and antigen before addition to the patient's serum. With a view to standardisation, preserved guinea-pig complement (Sonnenschein, I930 ; Green, I938) was used, and the cells were obtained from two sheep maintained in the animal house of the laboratory, and each bled on alternate weeks.

\section{The Meinicke Test}

This was the Meinicke-Klärungs reaction (M.K.R.II), $\dagger$ Meinicke and Grün (I932). The principle of the test is the mixing of 0.5 c.c. of two different preparations of the antigen with 0.2 c.c. and $0 \cdot 1$ c.c. respectively of unin-

* This work was done at the Liverpool City Laboratories during 1937 and 1938.

$\dagger$ The antigen used and a guide for the method may be obtained from Messrs. John Bell and Croyden, London. 
activated patient's serum, when, if the result be positive, flocculation, sedimentation and clarification take place slowly in the tubes. The strength of the reactions was determined by the degree of clarification after the mixtures had stood for eighteen hours in an incubator at $20^{\circ} \mathrm{C}$., and the results were read as follows :-

(I) No appreciable change in either tube

(opaque) $=-$ (negative).

(2) Marked clarification with $0 \cdot 2$ c.c. serum (opalescent) $= \pm$ (doubtful).

(3) Complete clarification with 0.2 c.c. serum (generally crystal clear) $=+$ (weak positive).

(4) Complete clarification with $0 \cdot 2$ serum and marked clarification with $0 \cdot$ I c.c. $=+$ (medium positive).

(5) Complete clarification in both tubes $=+$ (strong positive).

(6) More clarification with $0 \cdot$ I c.c. serum than with $0 \cdot 2$ c.c. serum (reversal of reaction)

$$
=+ \text { (very strong positive) }
$$

This interpretation of the results is a modification of the one proposed by Meinicke and Grün, and it was found useful to grade the Wassermann reactions somewhat similarly according to the amount of hæmolysis with 3 and 5 m.h.d. of complement, but correlation in strength with the M.K.R. II is not thereby implied.

\section{Procedure}

The sera for this investigation, with but few exceptions, were obtained from the Seamen's Dispensary and Mill Road Infirmary Venereal Diseases Clinics, Liverpool, and the tests were first compared in cases of registered or clinical syphilis. The number of sera received from individual patients varied, but in Tables I-III are shown the results with the first specimens from I,000 consecutive cases, consisting of 7 II males and 289 females, in which either or both tests gave a reaction other than negative.

It is seen in Table I that the W.R. was positive in 8I per cent. and the M.K.R. II in 93 per cent. of cases of syphilis. The tests agreed in 80 per cent. of cases. In Table II the results with the untreated and treated patients are considered separately. 


\section{THE WASSERMANN AND MEINICKE TESTS}

TABle I.-Comparison OF THE W.R. AND M.K.R. II IN I,OOO * CASES OF SYPHILIS

\begin{tabular}{|c|c|c|c|c|c|c|c|}
\hline \multirow{2}{*}{ Stage of Syphilis. } & \multirow{2}{*}{ No. of Cases. } & \multicolumn{3}{|c|}{ W.R. } & \multicolumn{3}{|c|}{ M.K.R. II. } \\
\hline & & + & \pm & - & + & \pm & - \\
\hline 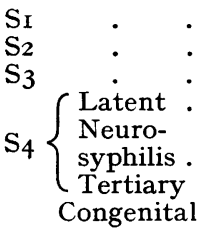 & $\begin{array}{r}150 \\
74 \\
40 \\
358 \\
\\
201 \\
83 \\
94\end{array}$ & $\begin{array}{r}115 \\
68 \\
35 \\
283 \\
\\
154 \\
74 \\
82\end{array}$ & $\begin{array}{r}5 \\
\mathrm{I} \\
\mathrm{I} \\
\mathrm{I} 2 \\
\mathrm{I} \\
\mathrm{I} 2 \\
\mathrm{I} \\
\mathrm{I}\end{array}$ & $\begin{array}{r}30 \\
5 \\
4 \\
63 \\
\\
35 \\
8 \\
11\end{array}$ & $\begin{array}{r}133 \\
72 \\
36 \\
331 \\
\\
187 \\
80 \\
92\end{array}$ & $\begin{array}{r}12 \\
2 \\
3 \\
24 \\
12 \\
2 \\
1\end{array}$ & $\begin{array}{l}5 \\
\text { I } \\
3 \\
2 \\
\text { I } \\
\text { I }\end{array}$ \\
\hline Total & $\mathrm{I}, \mathrm{OOO}$ & $8 \mathrm{II}$ & 33 & I 56 & 931 & 56 & 13 \\
\hline
\end{tabular}

* Including 4 patients classified in two stages of syphilis.

$S_{1}, S_{2}=$ primary and secondary syphilis.

$S_{3}=$ syphilis latent within one year of infection.

$S_{4} \quad=$ syphilis of longer duration than one year.

This classification is according to the Ministry of Health, London, but tertiary and neurosyphilis were considered separately.

TABLE II.-COMPARISON OF THE W.R. AND M.K.R. II in the Untreated and Treated Cases of Syphilis

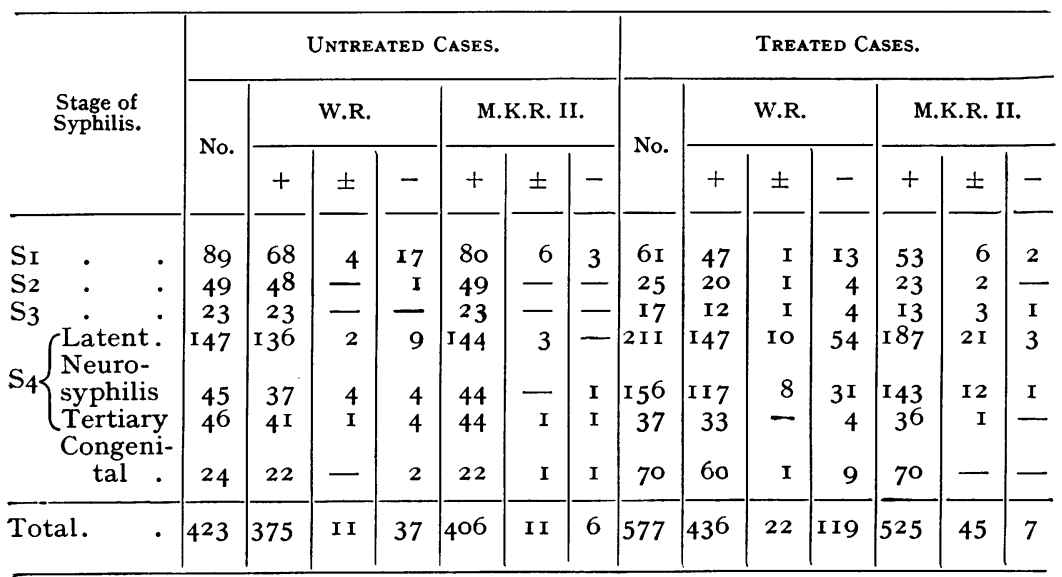


The W.R. and M.K.R. II agreed in untreated syphilis in 87 per cent. of cases and in treated in 73 per cent. The relative percentages of positive $(+)$ results in Table II are shown in the figure.

MKR.I. W.R.

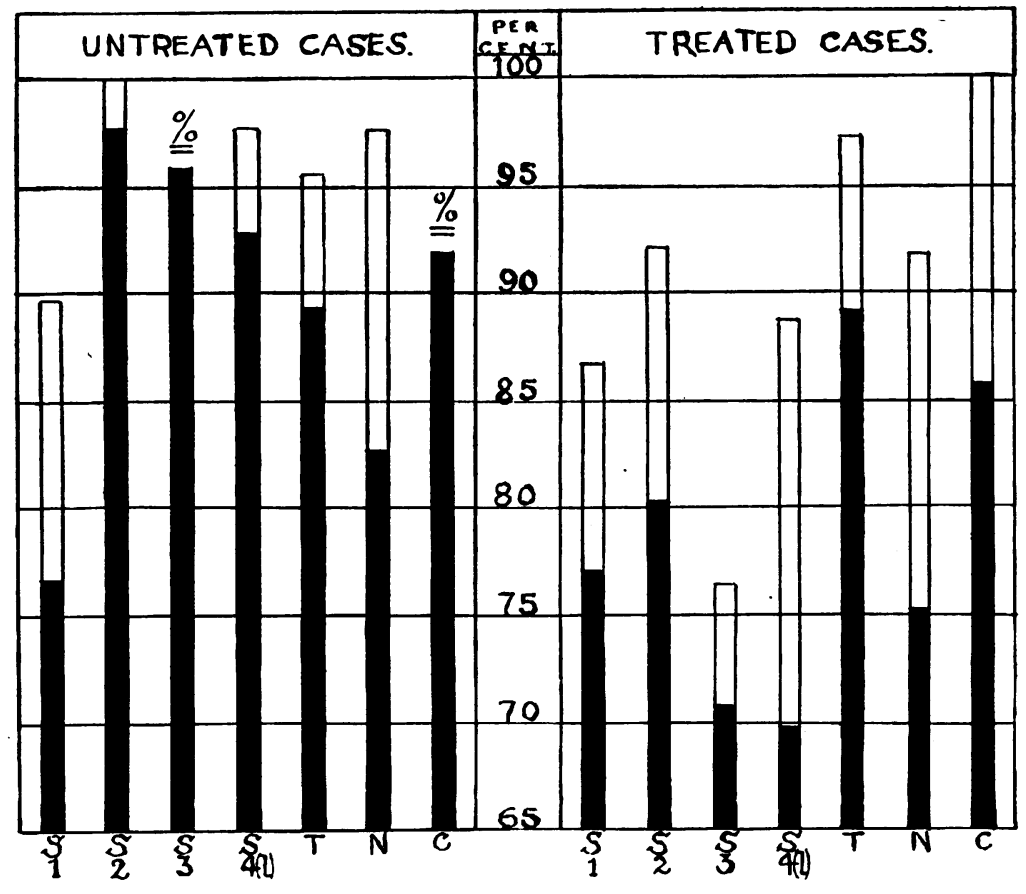

$\mathrm{T}=$ Tertiary. $\quad \mathrm{N}=\mathrm{NeURosy}$ Ph. $\quad \mathrm{C}=$ Congenital. $(l)$ latent. Relative percentages of positive results in untreated and treated syphilis.

In no stage of syphilis was the W.R. more sensitive than the M.K.R. II. The difference in the relative sensitivity of the tests is shown in Table III.

Table III.-Differences by Which Percentages Positive BY M.K.R. II EXCEEDED THOSE BY W.R.

\begin{tabular}{|c|c|c|c|c|c|c|c|c|}
\hline \multirow{2}{*}{ Classification. } & \multicolumn{8}{|c|}{ Stages of Syphilis. } \\
\hline & $\begin{array}{c}\text { Neuro- } \\
\text { syphilis. }\end{array}$ & $\underset{\text { (latent). }}{\mathrm{S}_{4}}$ & Sr. & $\begin{array}{l}\text { Cong. } \\
\text { syphilis. }\end{array}$ & Tertiary. & S2. & $\mathrm{s}_{3}$ & $\begin{array}{c}\text { All } \\
\text { stages. }\end{array}$ \\
\hline All Cases & $16 \cdot 4$ & 13.4 & $12 \cdot 0$ & $10 \cdot 6$ & $7 \cdot 2$ & $5 \cdot 4$ & $2 \cdot 5$ & $12 \cdot 0$ \\
\hline Untreated Cases & 15.6 & $5 \cdot 5$ & 13.5 & $0 \cdot 0$ & $6 \cdot 5$ & $2 \cdot 0$ & $0 \cdot 0$ & $7 \cdot 3$ \\
\hline Treated Cases & $16 \cdot 6$ & $18 \cdot 9$ & $9 \cdot 8$ & $14 \cdot 3$ & $8 \cdot \mathrm{I}$ & $\mathrm{r} 2 \cdot 0$ & $5 \cdot 9$ & 15.4 \\
\hline
\end{tabular}




\section{THE WASSERMANN AND MEINICKE TESTS}

It is seen in Table III that the difference between the two tests was greater in treated than in untreated syphilis except with SI cases. This exception is explained by the fact that the M.K.R. II was usually positive earlier than the W.R. in untreated primary syphilis. Complete agreement of the tests was found in $\mathrm{S}_{3}$ and congenital untreated patients and the greatest difference in neurosyphilis and congenital and $\mathrm{S}_{4}$ (latent) treated syphilis. The relatively close agreement found in $\mathrm{S}_{4}$ latent untreated patients may have been due to a diagnosis not being made for some with no clinical signs of syphilis in whom the M.K.R II was positive but the W.R. negative.

Because of this greater sensitivity of the M.K.R. II the results of comparative W.R. on sera from I99 patients made in collaboration with the Ministry of Health Laboratory, London, in 1936, are recorded (Table IV) in order to show the sensitivity of the Wassermann technique used in the present investigation.

Table IV.-Comparative Wassermann Tests

\begin{tabular}{|c|c|c|c|c|c|c|c|c|c|c|c|c|}
\hline \multirow{3}{*}{ Laboratory. } & \multicolumn{8}{|c|}{ CASES OF SyPhiLIs. } & \multirow{2}{*}{\multicolumn{4}{|c|}{$\begin{array}{l}\text { Cases with no evidence } \\
\text { or history of Syphilis. }\end{array}$}} \\
\hline & \multicolumn{4}{|c|}{ UNTREATED. } & \multicolumn{4}{|c|}{ TREATED. } & & & & \\
\hline & No. & + & \pm & - & No. & + & \pm & - & No. & + & \pm & - \\
\hline Ministry of Health & 3 & 3 & - & 一 & 105 & 19 & 22 & 64 & 91 & - & 12 & 79 \\
\hline City of Liverpool . & 3 & 3 & - & - & 105 & 25 & 2 & 78 & 9I & 3 & I & 87 \\
\hline
\end{tabular}

It is shown in this table that more positive results were obtained in the Liverpool laboratory from patients with syphilis. The many doubtful results in syphilis in the Ministry of Health laboratory may not be significant because I3 per cent. were also given by non-syphilitics. Three sera, including two from one patient at an interval of two weeks, were apparently non-specifically positive in Liverpool and doubtful in the Ministry of Health laboratory, one of these being positive there five months later, but in neither patient were there any clinical signs to support a diagnosis of syphilis. 


\section{BRITISH JOURNAL OF VENEREAL DISEASES}

It is thus evident that the Wassermann method used in the Liverpool laboratory on this comparison and standard was as sensitive as that used in the Ministry's laboratory.

In order to increase, if possible, the sensitivity of the W.R. I55 sera with which the M.K.R. II was positive or doubtful and the W.R. weaker or negative were re-tested and an additional and higher concentration of patient's serum $(I: 2)$ used in the W.R. The results obtained are recorded in Table $\mathrm{V}$.

(Note.-Higher concentrations of patients' serum were advocated by Wyler (I932) for increasing the sensitivity of the W.R. (No. I method), and although used in this comparison I have no evidence of the specificity of this procedure.)

TABle V.-Comparison OF THE W.R. AND M.K.R. II in Syphilis When USing a Higher Concentration of Patients' Serum in the W.R.

\begin{tabular}{|c|c|c|c|c|c|c|c|}
\hline \multirow{2}{*}{ No. of Sera. } & \multicolumn{2}{|c|}{ Original Results. } & \multicolumn{3}{|c|}{$\begin{array}{l}\text { Result of W.R. when using I : } 2 \\
\text { Concentration of Patients' Serum. }\end{array}$} & \multicolumn{2}{|c|}{$\begin{array}{l}\text { Percentage showing increase } \\
\text { in Strength of W.R. }\end{array}$} \\
\hline & M.K.R. II. & $\begin{array}{l}\text { W.R. }(x: 5 \\
\text { serum). }\end{array}$ & + & \pm & - & + & \pm \\
\hline $26^{*}$ & + & \pm & $2 \mathrm{I}$ & 5 & . & $80 \cdot 8$ & . \\
\hline 93 & + & - & 39 & I 7 & 37 & $4 \mathrm{I} \cdot 9$ & $18 \cdot 3$ \\
\hline 36 & \pm & - & 9 & 4 & 23 & $25 \cdot 0$ & $I I \cdot I$ \\
\hline
\end{tabular}

* Two sera gave weaker results in the W.R. when a higher concentration was used and have not been included.

In this table the 26 doubtful Wassermann results with the routine amount of serum (I:5) seem significant because $2 I$ were positive with $I: 2$ serum. It is noteworthy that the percentage increases in the strengths of the W.R. when a higher concentration of serum was used are in accord with the descending order of sensitivity in which the original results are arranged. 


\section{DisagreEMENT OF THE W.R. AND M.K.R. II}

\section{A. Untreated Syphilis}

Table VI.-Particulars of the Disagreement of THE W.R. AND M.K.R. II IN UNTREATED SI Patients.

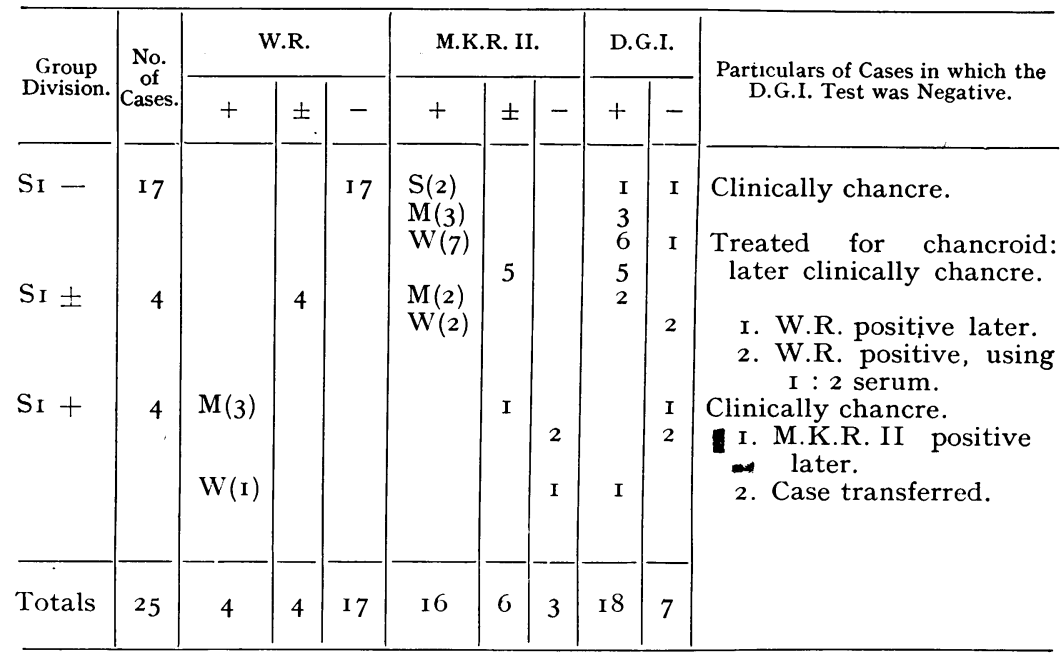

$\mathrm{SI}_{+}, \mathrm{SI}_{\mathrm{I}}=$ sero-positive and sero-negative primary syphilis, according to the result of the W.R., $\mathrm{S}_{I} \pm$ occupying an intermediate position (A. O. Ross).

$\mathrm{S}, \mathrm{M}, \mathrm{W}=$ strong, medium and weak positive (see interpretations of reactions under descriptions of methods used); the number of cases is in parenthesis.

D.G.I. = dark ground illumination; microscopical examination of serum exudate from the primary lesion for Spirochata pallida.

It is clear from this table that the results of the microscopical tests, all of which were made independently at the Seamen's Dispensary Clinic, confirm the greater sensitivity of the M.K.R. II.

\section{B. Treated Syphilis}

Seventy-one patients in whom the W.R. became and remained negative during treatment yielded positive results with the M.K.R. II six or more months later. In 28 of them the M.K.R. II was positive six to twelve months later, in 26 up to two years, in I3 up to three years and in 4 even longer. Of 35 sera which were negative by the routine W.R., I4 were positive and I 2 doubt- 
Table VII.-Particulars of the Disagreement of THE W.R. AND M.K.R. II IN UNTREATED SyPhILIS (continued)

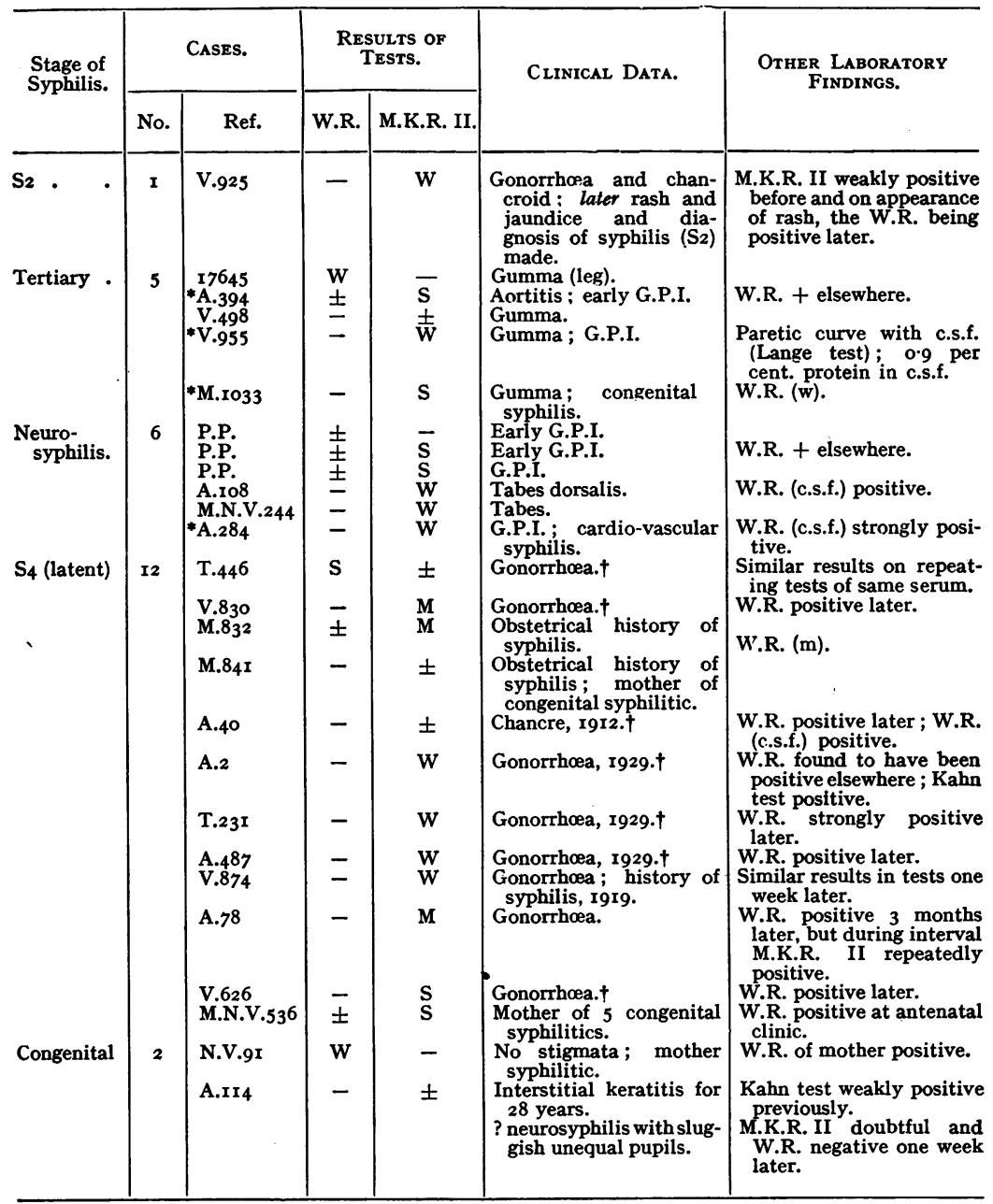

* Classifiable in more than one stage of syphilis.

$t$ These cases registered as latent syphilis on result of W.R. becoming positive or on behaviour of

reactions under repeated tests.
G.P.I. = general paralysis of the insane. P.P. = private practitioner. W.R. $(\mathrm{w}),(\mathrm{m})=$ weakly and G.P.I. $=$ general paralysis of the insane. P.P. $=$ private practitioner.
moderately positive W.R. with $\mathrm{I}: 2$ serum. C.s.f. = cerebrospinal fluid.

Other abbreviations as in preceding table.
Othe

ful when tested in a concentration of $I: 2$. The W.R. was positive in only two patients six or more months after the M.K.R. II remained negative. The persistence of positive Meinicke results was most noticeable in latent 


\section{THE WASSERMANN AND MEINICKE TESTS}

syphilis and in tabes dorsalis. In 40 patients with latent syphilis the M.K.R. II was positive and the W.R. negative, but nearly all of them had given a positive W.R. previously, the few exceptions being registered originally as sero-negative primary syphilis. The following results were found in neurosyphilis :-

TABle VIII.-COMPARISON OF THE W.R. AND M.K.R. II in Treated Neurosyphilis

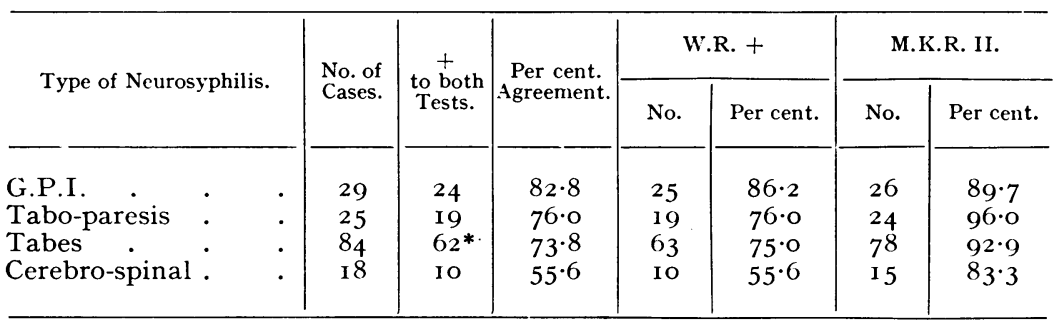

* Including I case \pm to both tests.

It appears from the results in this table that the closest agreement of the tests is in G.P.I., and that the W.R. is least sensitive in cerebrospinal syphilis.

The Relative Non-specificity of the W.R. and M.K.R.II. - Non-specific reactions have been considered for the purposes of this paper to be positive $(+)$ results occurring at least twice in either test, to exclude technical error, when there is no clinical or other indication of syphilis. One case has been included in which both tests were weakly positive but were later negative. Sixteen hundred cases of either gonorrhœea, chancroid or non-venereal disease were studied, and two or more specimens of sera from each patient tested, except from patients with no venereal disease in whom the W.R. and M.K.R. II were both negative. According to the system of notation recommended by the League of Nations Health Committee, \pm results are not to be held as diagnostic of syphilis, and therefore such reactions have not been included. Details of the non-specific reactions are shown in Tables IX and X. 
Table IX.-Particulars of the Non-SPecificity of THE W.R. AND M.K.R. II

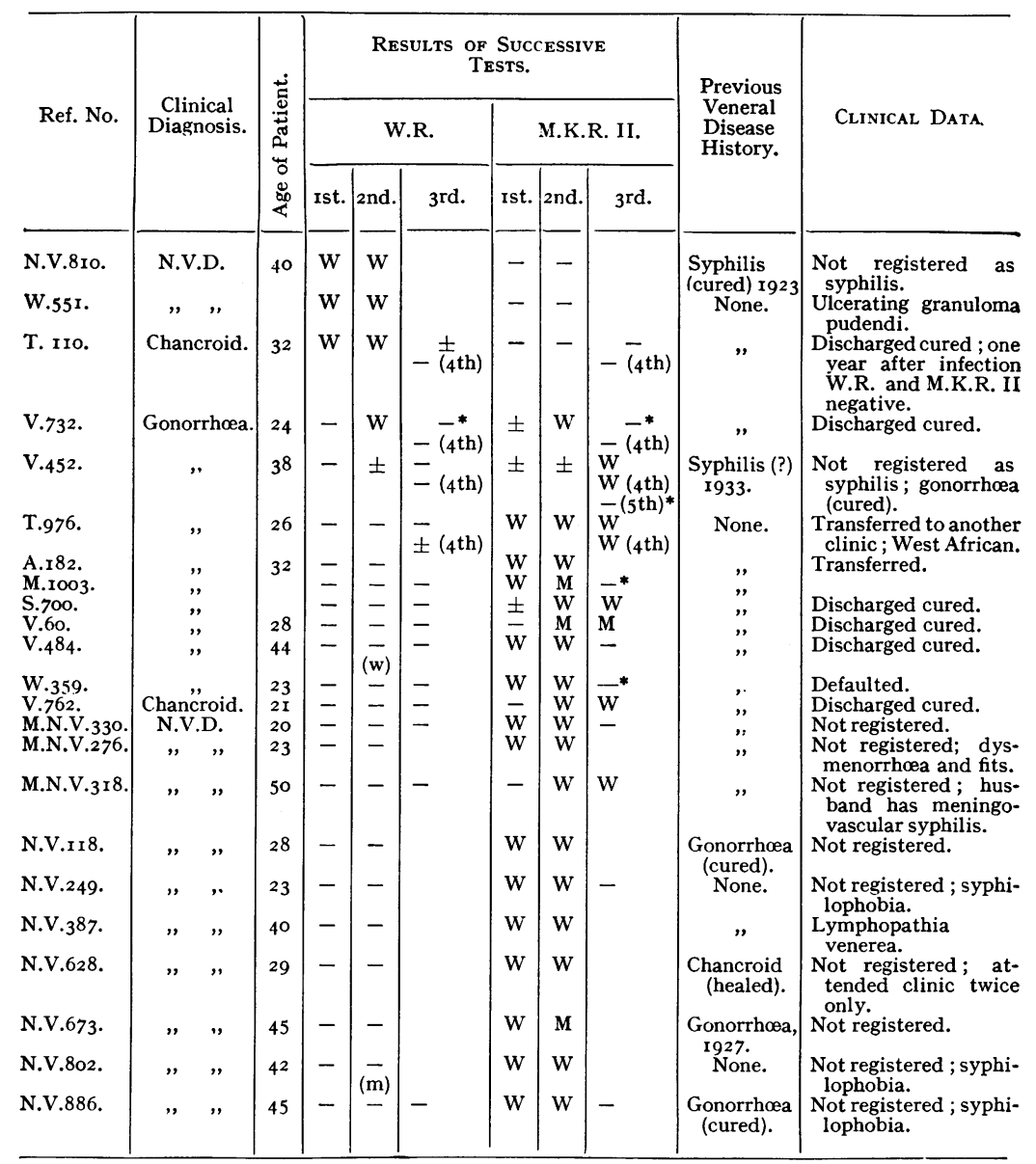

N.V.D. $=$ Non-venereal disease. $\quad *$ Negative after a provocative injertion of novarsenobillon. Other abbreviations as in Tables VI. and VII.

This non-specificity is condensed in Table X.

Thus in respect of sensitivity M.K.R. II gave I2 per cent. more positive reactions in syphilis (see Table I), and in respect of specificity only I per cent. more positive reactions in alleged non-syphilitic cases (Table X). 
THE WASSERMANN AND MEINICKE TESTS

Table X.-The Relative Non-specificity of the W.R. AND M.K.R. II

\begin{tabular}{|c|c|c|c|c|c|c|}
\hline \multirow{3}{*}{\multicolumn{2}{|c|}{ Clinical Condition. }} & \multirow{3}{*}{ No. of Cases. } & \multicolumn{4}{|c|}{ Non-specific Results } \\
\hline & & & \multicolumn{2}{|c|}{ No. Positive (+) } & \multicolumn{2}{|c|}{ Per cent. Positive. } \\
\hline & & & W.R. & M.K.R. II. & W.R. & M.K.R. II. \\
\hline Gonorrhœa & . & 660 & I & 9 & $\mathrm{O} \cdot \mathrm{I} 7$ & $I \cdot 36$ \\
\hline Chancroid & . & 36 & I & I & 0.03 & 0.03 \\
\hline N.V.D. . & . & 904 & 2 & IO & $0 \cdot 22$ & $I \cdot I I$ \\
\hline Total & . & $I, 600$ & 4 & 20 & $0 \cdot 25$ & $I \cdot 25$ \\
\hline
\end{tabular}

\section{Discussion}

The M.K.R. II appears to be little used in this country, and the present investigation was undertaken in order to determine if the test were suitable for use in parallel with the W.R. in view of the desirability of employing two serological methods in the diagnosis of syphilis. Prior to the present comparison, the Kahn test had been compared with the method of the W.R. employed here and had been found less sensitive, as is shown in Tables XI and XII.

TABle XI.-A Comparison of The W.R. AND KahN Test on Sera from Patients Attending the SEAMEN's Dispensary and Mill RoAd Infirmary Venereal Diseases Clinics, Liverpool.

\begin{tabular}{|c|c|c|c|c|c|c|c|c|c|}
\hline \multirow{2}{*}{ Description of Case. } & \multirow{2}{*}{$\begin{array}{c}\text { No. of } \\
\text { Patients. }\end{array}$} & \multicolumn{3}{|c|}{ W.R. } & \multicolumn{3}{|c|}{ Kahn Test. } & \multicolumn{2}{|c|}{ Percentage +} \\
\hline & & + & \pm & - & + & \pm & - & W.R. & $\begin{array}{c}\text { Kahn } \\
\text { Test. }\end{array}$ \\
\hline Syphilis (treated and & & & & & & & & & \\
\hline untreated) . & 359 & 183 & I 5 & 161 & 155 & 27 & I 77 & $50 \cdot 9$ & $43^{\cdot 2}$ \\
\hline Gonorrhœa & 136 & I & I & I 34 & . & 4 & I32 & 0.37 & \\
\hline Non-venereal disease & 131 & - & 7 . & 124 & I & 3 & $127\}$ & 3 & 3 \\
\hline
\end{tabular}

From this table it is seen that the tests were equal in specificity, but that the W.R. gave $7 \cdot 7$ per cent. more positive results in syphilitics. The difference of results in syphilis was as follows :- 
Table XII.-Relative Results with Sera with Which THE Tests Differed

\begin{tabular}{c|c|c|c|c|c|c|c}
\hline \multicolumn{2}{c|}{ W.R. } & \multicolumn{2}{c|}{ Kahn Test. } & \multicolumn{2}{c|}{ Kahn Test. } & \multicolumn{2}{c}{ W.R. } \\
\hline+ & + & + & - & + & + & + & - \\
& - & - & & & - & - & \\
\hline 32 & $\dot{8}$ & I2 & $\begin{array}{c}20 \\
8\end{array}$ & 4 & I0 & 2 & $\begin{array}{r}2 \\
\text { I0 }\end{array}$ \\
\hline
\end{tabular}

The technique of the Kahn test was as described by Kahn (I928) and the antigen was supplied by Parke Davis \& Co. and Burroughs Wellcome \& Co.

There have been many modifications of the Meinicke test, but the latest method, M.K.R. II, was used here, and is to be distinguished from an earlier one, M.K.R. (Meinicke, I929), in the references to literature that follow.

Before beginning this comparison a few hundred Meinicke tests were done to acquire the technique and the results of these have not been included in the series. Many others were also done that are not recorded, but these were mostly on sera from cases of treated syphilis and the results were negative with both M.K.R. II and W.R. As it is important in any comparison of this nature to know the sensitivity of the W.R., I have recorded in Table IV the results of a comparison of Wassermann tests made with the Ministry of Health Laboratory, London. Kolmer (I929) and Eagle (I937) claim that the W.R. is more sensitive when the ice-chest instead of the incubator is used to fix guinea-pig complement. Two hundred parallel Wassermann tests were therefore done by the routine method and by one which differed only in that complement was allowed to fix for sixteen hours in the ice-chest, followed by fifteen minutes at $37^{\circ} \mathrm{C}$., and these were compared with the M.K.R. II. The routine method consists of fixation for thirty minutes at room temperature followed by thirty minutes at $37^{\circ} \mathrm{C}$. in the water bath.

There was no great difference in the clear-cut reactions of the parallel Wassermann tests, but with 2 cases of syphilis the M.K.R. II was positive and the routine W.R. negative, and I of these was negative when ice-chest 
fixation was used. The W.R. seemed less specific with ice-chest fixation and many doubtful reactions were obtained, and occasionally the results were anti-complementary although a preserved complement was used. However, the technique employed may not have been suitable for this method of complement fixation or the preserved complement may have deteriorated in the dilution used after prolonged incubation at low temperature (Eagle).

Harrison (I93I) quoted Wyler (unpublished), who found the No. I W.R. method of the Medical Research Committee slightly more sensitive than the Wassermann test of Kolmer.

Whenever possible the positive Meinicke results were evaluated by additional tests, more particularly by using a higher concentration of patient's serum in the W.R. This procedure is now used regularly in the Liverpool laboratory when the results of both tests are not in relative agreement, and seems to enhance the sensitivity of the W.R.

An important finding in this investigation is the higher sensitivity of the M.K.R. II in most stages of syphilis.

With regard to the results as a whole, Mr. Caradoc Jones, statistician Liverpool University, has very kindly furnished the following comments :-

“ I. B test (M.K.R. II) has 23 more cases than A test (W.R.) out of $\mathrm{r}, 000$ classed as doubtful. This difference may be significant but is not markedly so.

2. Omitting the doubtful cases (quoted from Table I) M.K.R. II gives $98 \cdot 6 \%+$ results

W.R. , $83.9 \%$ + results a difference of $14.7 \%$.

The standard error of this difference is only $\mathrm{I} \cdot 2 \%$ so that the difference of + results is certainly significant, i.e., it cannot be explained by random sampling."

3. On the other hand the M.K.R. II indicates syphilis in $I \%$ more cases than the W.R. (Table X) where there is no clinical evidence of syphilis or history of it in the patients. It is unlikely that this difference is due only to random sampling (standard error $=0 \cdot 3 \%$ )." 
The differences in sensitivity in different stages of syphilis were as shown below.

\section{A. Untreated Syphilis}

The higher sensitivity of M.K.R. II was very evident in the primary stage before the W.R. became positive and should be an aid to early diagnosis. With tertiary syphilis the W.R. was positive in one patient when the M.K.R. II was negative. This happened in a man with a gummatous ulceration of the leg, but in three other patients with gummata the W.R. was negative and the M.K.R. II positive in two and doubtful in one. In latent syphilis (S4) the M.K.R. II yielded 5.5 per cent. more positive results than the W.R., but this difference might have been greater with a higher evaluation of weakly positive Meinicke results in the absence of clinical findings. Such results were significant in primary syphilis (Table VI) for Io patients with negative W.R. and positive M.K.R. II (including 6 weakly positive results) showed the presence of Spirochata pallida in the primary lesions. Most of these latent $\mathrm{S}_{4}$ patients, however, in whom the M.K.R. II but not the W.R. was positive were only registered as syphilis when the W.R. became positive later. With neurosyphilis the M.K.R. II was positive in 44 out of 45 patients and the W.R. positive in 37 , the difference of results being found in 5 patients with G.P.I. and 2 with tabes dorsalis. The W.R. was doubtful in 3 and negative in 2 of the former patients and negative in both of the latter. In untreated syphilis as a whole the M.K.R. II gave $7 \cdot 3$ per cent. more positive results than the W.R.

\section{B. Treated Syphilis}

With neurosyphilis the percentages in which the M.K.R. II was returned more frequently positive were 27.7 (cerebrospinal), 20.0 (tabo-paresis), I7.9 (tabes) and 3.5 (G.P.I.). The relatively close agreement for G.P.I. is interesting because Ogden and Partner (I93I) found the W.R. positive and the M.K.R. negative in 9 patients with syphilis, including 7 under treatment, and all had G.P.I. It is seen above, however, that I found no such close correlation of the results of the tests in untreated patients with this condition, in fact the highest sensitivity of the M.K.R. II with neurosyphilis was shown in G.P.I. 
The M.K.R. II was much more sensitive than the W.R. in congenital syphilis, but the difficulty of effecting a cure in this condition is well known. The M.K.R. II was also much more sensitive in cerebrospinal cases, and this test, therefore, may be of value in the diagnosis of obscure hemiplegia when the W.R. of the blood and cerebrospinal fluid are negative in old treated syphilis. The cerebrospinal fluid as well as the blood may give a negative W.R. when a flocculation test is more sensitive but positive only with the blood (Stern and Frank, I932).

The 18.9 per cent. higher sensitivity of the M.K.R. II in $\mathrm{S}_{4}$ (latent) cases is in marked contrast to the figure of 5.5 per cent. given for the untreated patients in this group. The difference in sensitivity in treated syphilis was more than twice that found in untreated syphilis. The tests agreed in 80 per cent. of all cases of syphilis, and this compares well with the 84 per cent. agreement obtained by Ogden and Partner. Loewy (I929) found that the M.K.R. gave 8.6 per cent. more positive results than the W.R. in patients with past histories of syphilis, and in none was the reaction positive in the absence of syphilis. Reichel (1930) tested 588 sera from known syphilitics, including many under treatment, and obtained 65.1 per cent. positive results with the M.K.R. but only $3 \mathrm{I}^{\circ} 99$ per cent. with the W.R., a difference of $33 \cdot I I$. He concluded from the examination of 4I2 sera from non-syphilitics that the M.K.R. gave 3.4 per cent. of non-specific results. I got I2 per cent. more positive results with the M.K.R. II than with the W.R. in syphilis and found the apparent non-specificity of the former test to be $\mathrm{I} \cdot 25$ per cent. ; thus the M.K.R. used by Reichel was relatively nearly three times as sensitive as the M.K.R. II but equivalently less specific.

Stern and Frank compared the M.K.R. with three other tests in the examination of 3,678 sera from syphilitics and non-syphilitics and found that the sensitivity of these tests in descending order was Müller II (conglobation reaction), M.K.R., W.R. and Kahn, and the order of specificity, W.R., M.K.R., Kahn and Müller II. In the present series the M.K.R. II seemed less specific than the W.R., but this non-specificity, which was often presumptive, was not high and was found chiefly in seamen. It is seen in Table VII that 6 patients with negative W.R. and positive M.K.R. II, and suffering from gonorrhœa 
but with no history of syphilis, were registered as syphilis (S4), but only when the W.R. was positive later or found to have been positive elsewhere, and it is thus feasible that some of the patients with gonorrhœa who gave apparent non-specific Meinicke results (Table IX) had latent syphilis and may have given a positive W.R. if they had been followed up further. The M.K.R. II was non-specifically positive in the three patients with syphilophobia but with no signs of syphilis, in one patient whose husband had meningo-vascular syphilis and in another who had a history of suspected infection. The following two instances in which the Meinicke results were thought at first to be non-specific are quoted. V.925 (Table VII), originally registered as a case of gonorrhœea and chancroid, had a weakly positive M.K.R. II but the Wassermann was negative, and in the sore which was thought to be non-syphilitic no spirochætes were found; later the patient developed a secondary syphilitic rash and the blood W.R. was eventually returned positive. In Table VI a patient with a weakly positive M.K.R. II and a negative W.R. was registered as a case of chancroid, but later the appearance of the ulcer was typically that of chancre and the epitrochlear glands became palpable. The M.K.R. II was now moderately positive but the W.R. negative. No spirochætes were found in the primary lesion, probably on account of carbol-fuchsin local treatment during the original diagnosis of chancroid.

In Table $V$ there are 36 cases of syphilis with doubtful Meinicke and negative Wassermann results, and with only 13 of these was it found possible to change the W.R. to + or \pm by using a higher concentration of patients' serum, although in some instances fixation of complement may have been inhibited by this increased amount of serum (Eagle), and it is possible that these doubtful Meinicke results are not of much significance. One of the four patients with apparent non-specifically positive W.R. (Table IX) had a history of syphilis sixteen years previously, and was possibly a case of latent syphilis. Another patient was suffering from ulcerating granuloma pudendi, but a weakly positive W.R. may be found in this condition.

It is evident from the findings herein that the M.K.R. II is a valuable test for the diagnosis of syphilis, but of equal importance are the ease with which the results can be 


\section{THE WASSERMANN AND MEINICKE TESTS}

read, the simplicity of technique, and the saving of time and material in doing the tests. Thirty sera can be put up with antigen in about thirty minutes, and this fact offsets the possible disadvantage of the delay of overnight incubation. No Meinicke tests were done on cerebrospinal fluid.

\section{SUMMARY}

(I) A comparison of the M.K.R. II and W.R. in syphilis shows that the former is much more sensitive, especially in treated cases.

(2) In untreated patients this higher sensitivity is very evident with primary and neurosyphilis, and in the treated with latent, congenital and neurosyphilis.

(3) This difference in sensitivity may be lessened by increasing the concentration of patients' serum in the W.R.

(4) The M.K.R. II may be less specific than the W.R. but its non-specificity is low.

(5) The M.K.R. II is a valuable test to use in parallel with the W.R. for the serological diagnosis of syphilis.

I wish to express my thanks to Dr. Ross and the staffs of the Seamen's Dispensary and Mill Road Infirmary Clinics, Liverpool, for generous assistance in going through the records of many patients; to Mr. E. A. Hewish for technical assistance in part ; and to Professor H. D. Wright for helpful criticism.

\section{ADDENDUM}

Note on the M.K.R. II and the results obtained in the above comparison by $A$. O. Ross.

The Wassermann serum test has remained, in this country at least, the standard test for the presence of syphilis. Yet it cannot be denied that its reliability is found principally in the secondary stage of syphilis, and by the secondary stage of syphilis I mean that stage during which the patient is infectious through his or her natural mucous secretions. Before the advent of this stage and after it has passed active syphilis may be present and yet the W.R. is negative. Therefore it has become a commonplace to assert that a negative Wassermann report on a patient's serum simply means that the 
patient has a negative W.R., and unsupported by other evidence is not a diagnostic criterion. One has only to recall the serological reactions of tabes dorsalis to realise the truth of this assertion, for in quite 25-30 per cent. of such cases the W.R. is reported negative. Again it is a common occurrence for the W.R. in latent syphilis to vary from week to week, and the whole range of reports from negative to strongly positive may be collected in three months without the assistance of so-called provocative tests or tentative treatment.

It has been the hope of the clinician that it would be possible to evolve some test, complementary to the W.R., more delicate and more constant and yet equally specific, for we are indeed grateful for the reliable positive W.R. It is comparatively easy to provide evidence that a test is more delicate and more constant than the W.R., but exceedingly difficult to convince those to whom the W.R. has been "a cloud by day and a pillar of fire by night", for well-nigh thirty years that the positivity of serum to some new test does mean syphilis. Of these conservatives I am one, because I cannot feel justified in treating for five years an apparently healthy person with no history of syphilis on the ground that his serum reacts positively to a new precipitation test. Yet I have before me the case of M.N.V. 3I8 in Table IX (non-specific reactions), who is aged fifty and has been married for thirty-one years. Her husband is now in hospital suffering from syphilitic hemiplegia, and it is probable that she has been infected at some time in these thirty-one years of married life. One must compare her case with M.84I and M.N.V.536 (Table VII), whose infection was proved by the fact that their children were congenital syphilitics, and with A.487, A.78, T.23I, V.626 and V.830 whose Wassermann tests were ultimately returned positive. Had it not been for this supporting evidence these latter 5 cases would have been grouped as non-specific reactors. There are other patients also in whom positivity of the M.K.R. II brought about a more intensive search for neurosyphilis with ultimate corroborative evidence of this condition (V.955, M.N.V.244, A.Io8, etc., in Table VII). It is noteworthy also that in I7 cases of primary syphilis with negative W.R: the M.K.R. II was positive or doubtful, and in 15 of them Spirocheta pallida was found in the primary ulcer. One of the other two patients did not 


\section{THE WASSERMANN AND MEINICKE TESTS}

remain long enough for dark ground tests to be repeated, and in one the ulcer was diagnosed as chancroid at first and treated accordingly.

With regard to Table IX generally, which is concerned with non-specificity, several patients with negative W.R. and weakly positive M.K.R. II were suffering from syphilophobia, and in the absence of definite history and signs of syphilis it was thought to be psychologically unsound to follow these up further. Others, mostly seamen and some of them aliens or coloured, it was difficult to prevent from returning to employment at sea, and therefore tests could not be repeated and investigation of the cerebrospinal fluid, etc., performed. I thus feel that the figures of non-specificity for the M.K.R. II in Table X are thereby exaggerated.

Of the value of the M.K.R. II from the aspect of sensitivity it seems unnecessary for me to speak. In neurosyphilis I99 cases out of 201 were picked out by the M.K.R. II as against I 66 by the W.R. In treated syphilis from the clinician's point of view the M.K.R. II is excellent, more especially for those who are given to treating syphilis only until a negative W.R. is obtained, because, if they will take note of the result of the M.K.R. II, treatment will be continued greatly to the benefit of the patient. This lag in the reversal time of the M.K.R. II in treated syphilis is well demonstrated in primary cases, for this test remained positive while the W.R. was returned as negative in 9.8 per cent.; in secondary syphilis the corresponding figure was I2 per cent. In early cases chemotherapeutic treatment would appear to impede the natural immunological response of the patient, and it is essential that such treatment should be adequate to ensure biological cure and not only a " serological cure," which is so often temporary and leads to infectious relapse later.

To sum up, the M.K.R. II seems to be an excellent test for use in parallel with the routine W.R., since its non-specificity is slight and its sensitivity is such that it reveals the case which has not been adequately treated and leads to biological cure. The M.K.R. II is also of valuable assistance in the diagnosis of early primary and late syphilis, and especially of neurosyphilis. During the months in which this investigation has progressed I have become increasingly impressed as a clinician with the 
diagnostic and directive value of the M.K.R. II in the conduct of treatment.

\section{REFERENCES}

EAGLE, H. (I937) : “The Laboratory Diagnosis of Syphilis," London.

GreEN, G. A. (I938) : "Preservation of Complement for the Wassermann Reaction," Jour. Path. \& Bact., 46, 382.

HARRISON, L. W. (I93I) : "A System of Bacteriology in Relation to Medicine," 8, 250, H.M. Stationery Office.

KaHN, R. L. (I928) : "The Kahn Test" (A practical guide), pp. IIII3I, London.

Kolmer, J. A. (I929) : "Serum Diagnosis by Complement Fixation," London.

LoEwy, E. (I929): "Erfahrungen mit der neuen Meinickeschen Klärungsreaktion (M.K.R.)," Dissertation Univ. Koln.

MeINICKE, E. (I929): "Meine Klärungsreaktion auf Syphilis (M.K.R.)," Munch. med. Wochr., 76, I965.

MeINICKE, E., and GRÜN, E. (I932) : "Meine neue Klärungsreaktion auf Syphilis" (Meinicke-Klärungs Reaktion II, M.K.R. II). $Z$ bl. Bakt., 125, 356.

Ogden, W., and Partner, F. (I93I): "The Meinicke Clarification Reaction for Syphilis in Mental Hospital Practice." Lancet, ii., I2I.

Reichel, H. J. (I930) : “Die Meinicke-Klärungsreaktion auf Syphilis," Zbl. Bakt., 116 (4/5), 349-352.

SonNenschein, C. (I930): Ztschr. ImmunForsch., 67, $5 \mathrm{I} 2$.

STERN, M., and FraNK, T. (I932) : "Der Vergleichswert der Wassermann-Reaktion speziell mit Wadsworth-Extrakten, und der KahnMeinicke Klärung-und Müller Ballung II-Reaktion auf Grund von Paralleluntersuchungen mit 3678 seren," Arch. Dermatol. u. Syph., 166 (1), pp. 76-84.

Wyler, E. J. (I929) : Medical Research Committee, " Special Report Series No. I29," H.M. Stationery Office ; (I932) : " Report on Public Health and Medical Subjects No. 67," Ministry of Health, London. 